\title{
Prediction of spatio-temporal bone formation in scaffold by diffusion equation
}

\author{
Alireza Roshan-Ghias ${ }^{a}$, Arne Vogel $^{a}$, Lalaonirina Rakotomanana ${ }^{b}$, Dominique P. Pioletti ${ }^{\mathrm{a}, *}$ \\ a Laboratory of Biomechanical Orthopedics, Ecole Polytechnique Fédérale de Lausanne, Switzerland \\ ${ }^{\mathrm{b}}$ IRMAR, University of Rennes I, 35042 Rennes Cedex, France
}

\section{A R T I C L E I N F O}

\section{Article history:}

Received 13 April 2011

Accepted 28 May 2011

Available online 23 June 2011

\section{Keywords:}

Mathematical modeling

Bone formation

Animal model

Micro-CT imaging

\begin{abstract}
A B S T R A C T
Developing a successful bone tissue engineering strategy entails translation of experimental findings to clinical needs. A major leap forward toward this goal is developing a quantitative tool to predict spatial and temporal bone formation in scaffold. We hypothesized that bone formation in scaffold follows diffusion phenomenon. Subsequently, we developed an analytical formulation for bone formation, which had only three unknown parameters: $C$, the final bone volume fraction, $\alpha$, the so-called scaffold osteoconduction coefficient, and $h$, the so-called peri-scaffold osteoinduction coefficient. The three parameters were estimated by identifying the model within vivo data of polymeric scaffolds implanted in the femoral condyle of rats. In vivo data were obtained by longitudinal micro-CT scanning of the animals. Having identified the three parameters, we used the model to predict the course of bone formation in two previously published in vivo studies. We found the predicted values to be consistent with the experimental ones. Bone formation into a scaffold can then adequately be described through diffusion phenomenon. This model allowed us to spatially and temporally predict the outcome of tissue engineering scaffolds with only 3 physically relevant parameters.
\end{abstract}

(c) 2011 Elsevier Ltd. All rights reserved.

\section{Introduction}

Bone formation inside scaffold is a complex process. It involves migration of several cell types [1], proliferation [2], differentiation [3], angiogenesis [4,5], and mineralization [6], as well as the interaction of numerous cytokines and growth factors [7]. Bone formation also depends on the architecture of the scaffold [8], its surface properties [9], the mechanical stimulation [10,11], the implantation site [12], and the bone-scaffold interface [13]. Probing the effects of each parameter and their interaction has proven challenging. Mathematical modeling of bone formation inside scaffolds has been used to understand the underlying mechanisms and help design experiments.

The existing mathematical models are mainly based on mechano-regulation theories [14-16], or growth factor dynamics $[17,18]$, similar to computational studies on fracture healing $[7,19,20]$. These models allow studying the effect of scaffold architecture, cell density, mechanical stimulation and vascularization on the fate of tissue engineering constructs. The available models are usually complex with several coupled nonlinear equations, involving dozens of unknown parameters [18]. The theoretical

\footnotetext{
* Corresponding author. EPFL/STI/IBI/LBO, Station 15, 1015 Lausanne, Switzerland. Tel.: +4121693 8341; fax: +41216938660.

E-mail address: dominique.pioletti@epfl.ch (D.P. Pioletti).
}

studies are mostly developed without accompanying experiments to identify the numerous parameters appearing in the models. The results are then compared qualitatively with experimental observations [7].

In the present study, we take an alternative approach. We hypothesize that bone formation in scaffold follows the diffusion equation. To test the hypothesis, a longitudinal in vivo experiment is developed to spatially and temporally quantify osteogenesis inside scaffold using micro-computed tomography. The unknown parameters of the model are identified with the in vivo data. The model having only 3 identified interpretable parameters is then used to predict bone formation reported in two published in vivo experiments.

\section{Materials and methods}

\subsection{Surgery}

Left distal femurs of 7 female Wistar rats (weight 245-250 gr) were drilled (Veterinary Authority from the Canton of Vaud, authorizations No. 2140) and scaffolds of $3 \mathrm{~mm}$ in diameter and height made of PLA $+5 \%$ wt. $\beta$-TCP [21] were implanted inside the holes (Fig. 1a) following a published protocol [11]. Prior to implantation, the scaffolds were perfused with PBS to remove air bubbles. No cells or growth factors were added in the scaffold.

\subsection{Micro-CT scanning}

All animals were scanned at 7 time points $(2,4,7,11,15,22$ and 35 weeks after surgery) using SkyScan 1076 In Vivo Scanner (SkyScan, Belgium). Each leg was 


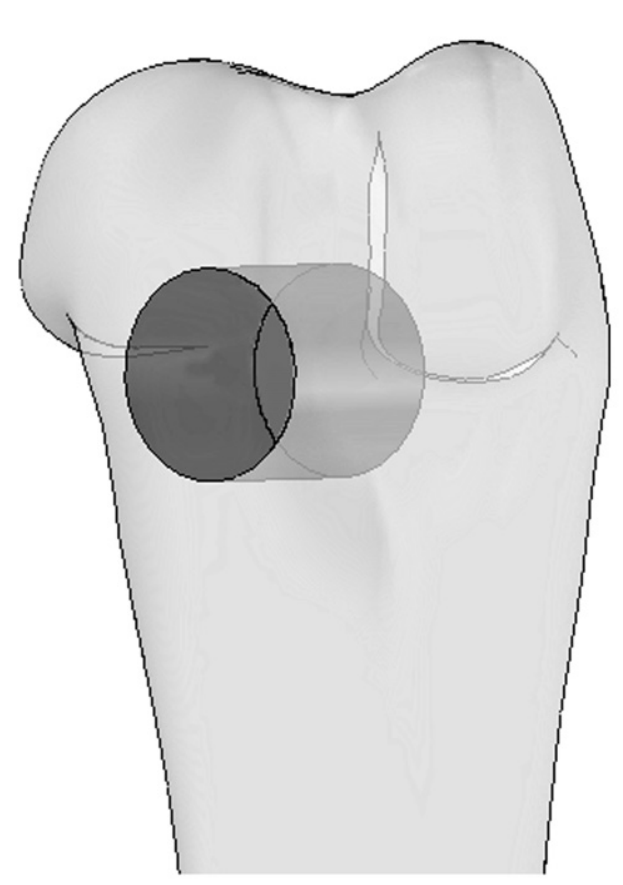

a

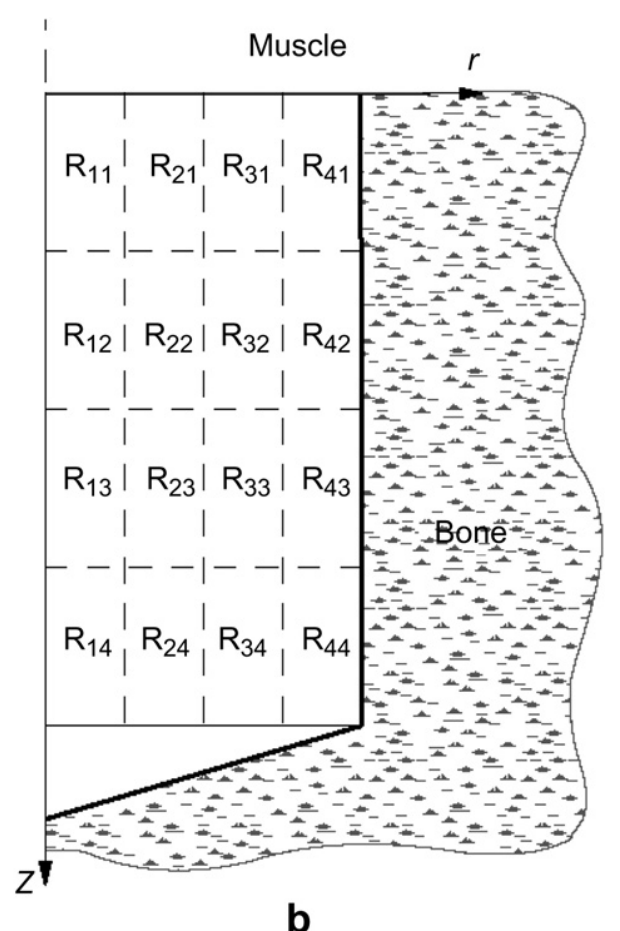

b

Fig. 1. (a) Anterior-posterior view of distal femur with an implanted scaffold, (b) the axisymmetric cross-section of bone and scaffold. The region of interest is a cylinder starting from the bone edge and ending at $1 \mathrm{~mm}$ from the end of the hole (the mark made by the drill tip).

stretched and separated from the body and scanned along with two phantoms and a tube of water for calibration purposes. The scanning parameters were the same for all scans ( $18 \mu \mathrm{m}, 80 \mathrm{kV}, 124 \mu \mathrm{A}, 1 \mathrm{~mm}$ Al filter, $600 \mathrm{~ms}$ exposure time). Reconstruction was done using NRecon software (SkyScan).

\subsection{Measurement of bone volume}

The region of interest (ROI) was a cylinder fitting the defect in the bone. It started from the exterior of the cortical bone and ended at $1 \mathrm{~mm}$ from the tip of the cone-shape defect made by the drill tip (Fig. 1b). The ROI was divided into 16 axisymmetric regions, such that the height and thickness of the regions were equal, resulting in 16 concentric regions. The images inside each region were segmented with a threshold of bone mineral density (BMD) equal to $0.5 \mathrm{gr} / \mathrm{cm}^{3}$. The corresponding bone volume fraction (BVF) in each region was quantified. All calculations were done in Matlab (Mathworks, USA).

\subsection{Mathematical model}

Assuming that the continuum model governing bone formation inside the scaffold was the diffusion equation, we have

$\frac{\partial c}{\partial t}=\alpha \Delta c$

where $c$ denoted the BVF, $t$ was time in days, $\alpha\left(\mathrm{mm}^{2} /\right.$ day) was defined as the socalled scaffold osteoconduction coefficient, and $\Delta$ was the Laplacian. Considering axisymmetric geometry, Equation (1) was written as

$\frac{\partial c}{\partial t}=\alpha\left[\frac{\partial^{2} c}{\partial r^{2}}+\frac{1 \partial c}{r \partial r}+\frac{\partial^{2} c}{\partial z^{2}}\right]$

where $r$ and $z$ were spatial variables of the coordinate system shown in Fig. 1b.

As BVF was higher in the surrounding bone tissue, we assumed that a bone flux exists from surrounding bone to scaffold, but that there was no flux at the scaffold-muscle interface. These hypotheses translated themselves in the following boundary conditions:

$$
\begin{gathered}
-\left.\alpha \frac{\partial c}{\partial r}\right|_{r=R}=h[C-c(r, z, t)]_{r=R} \\
\left.\frac{\partial c}{\partial r}\right|_{r=0}=0 \\
-\left.\alpha \frac{\partial c}{\partial z}\right|_{z=H}=h[C-c(r, z, t)]_{z=H} \\
\left.\frac{\partial c}{\partial z}\right|_{z=0}=0
\end{gathered}
$$

where $R$ and $H$ were the radius and height of the scaffold, respectively. We defined $h$ ( $\mathrm{mm} /$ day) as the so-called peri-scaffold osteoinduction coefficient, and $C$ as the final $B V F$. As the scaffold was initially devoid of bone, the corresponding initial condition was

$\left.c(r, z, t)\right|_{t=0}=0$

Employing the method of separation of variables, we solved Equation (2) with the defined boundary and initial conditions. The detail of the solution is given in the Appendix. Integrating over each region of interest inside the scaffold ( $R_{p q}$ in Fig. 1b), we obtained the bone volume (BV) in each region $R_{p q}$

$\left.B V(t)\right|_{R_{p q}}=\int_{V_{R_{p q}}} c(r, z, t) d V=C\left[V_{R_{p q}}-\sum_{i=1}^{\infty} \sum_{j=1}^{\infty} E_{i j} I_{i j} e^{-\left(r_{i}^{2}+\beta^{2} \omega_{j}^{2}\right) F o}\right]$

with

$E_{i j}=-\frac{4}{\gamma_{i}} \cdot \frac{B i}{\gamma_{i} J_{0}\left(\gamma_{i}\right)+B i \cdot J_{1}\left(\gamma_{i}\right)} \cdot \frac{\beta \sin \left(\omega_{j}\right) \tan \left(\omega_{j}\right)}{B i+\beta \sin ^{2}\left(\omega_{j}\right)}$

$I_{i j}=\frac{2 \pi R H}{\gamma_{i} \omega_{j}} \cdot\left[r J_{1}\left(\frac{\gamma_{i}}{R} r\right)\right]_{r_{p}}^{r_{p+1}} \cdot\left[\sin \left(\frac{\omega_{j}}{H} z\right)\right]_{z_{q}}^{z_{q+1}}$

Following an approach similar to heat transfer [30], we defined Bi and $F o$ as Biot and Fourier numbers, respectively

$B i=\frac{h R}{\alpha}, \quad F o=\frac{\alpha t}{R^{2}}$

The definition of other parameters is given in the Appendix. It is noteworthy to mention that although the equations seem to be complex, they contain only 3 unknown parameters, $C, \alpha$ and $h$.

\subsection{Model identification}

In order to have a finite series from Equation (6), $E_{i j}$ and $I_{i j}$ were calculated for successive values of $i$ and $j$. Both values converged rapidly, so they were truncated by the first five coefficients $(i, j=1,2,3,4,5)$. Nonlinear regression using the Levenberg-Marquardt algorithm [22] was employed to fit the in vivo data to the model and the three unknown parameters, $C, h$ and $\alpha$, were estimated. The sensitivity of the response to initial estimates for the parameter estimation was examined to test the uniqueness of the results. Confidence intervals of the estimated parameters were calculated using residuals and covariance matrix [22]. 


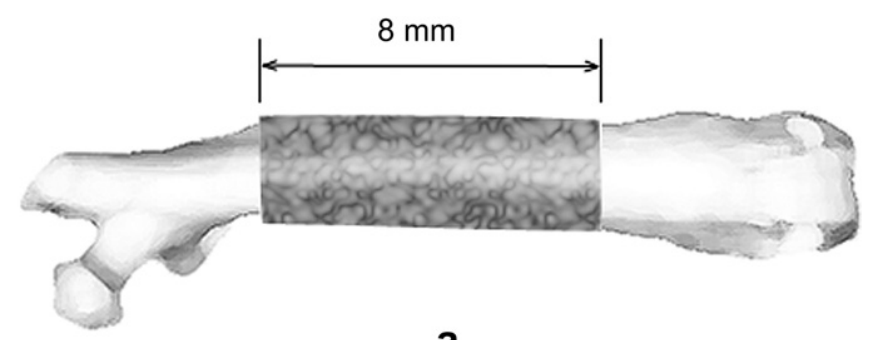

a

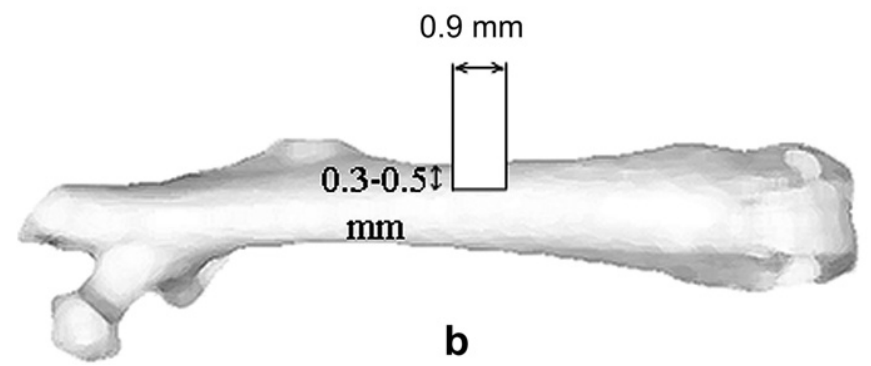

Fig. 2. (a) Segmental defect in rat femur [23], (b) cortical perforation in mouse femur [24].

\subsection{Parametric study}

One advantage of mathematical modeling is the ability to study the relationship between different parameters. Dimensional analysis, in particular, is a powerful technique that groups different parameters into fewer dimensionless numbers. Here, we have two dimensionless numbers: Biot and Fourier numbers ( $B i$ and Fo, respectively). We define the characteristic time, $T$, as the time needed for the scaffold to be filled with $75 \%$ of final BVF. A relation between $B i$ and Fo can be found by solving Equation (6) at $t=T$.

\subsection{Case studies}

The proposed model with estimated parameters, i.e. $C, h$ and $\alpha$ obtained from the present in vivo study, was used to predict the bone formation obtained from two other published in vivo experiments. The first in vivo experiment studied was a segmental bone defect in the rat femur [23], and the second one was cortical bone perforations in the mice femur [24].

In the first in vivo study used to validate our model, Rai et al. [23] implanted cylindrical polycaprolactone-20\% tricalcium phosphate (PCL-TCP) scaffolds in a rat femoral segmental defect. The scaffolds were $8 \mathrm{~mm}$ in height and $4 \mathrm{~mm}$ in diameter (Fig. 2a). The BVF were measured at 3 and 12 weeks post-operatively using a microCT. To identify the in vivo segmental defect with our model, we considered that bone flux enters the scaffold from the two ends only. The calculation of BVF with our model was implemented in COMSOL (Comsol Inc, USA) using axisymmetric geometry. The proximal and distal sides of the scaffold were subjected to flux boundary conditions. Zero flux condition was assigned to the exterior side of the scaffold. The model was meshed using triangular elements and the diffusion equation was solved.

In the second in vivo study used to validate our model, Monfoulet et al. [24] reported the pattern of bone healing in the cortical diaphysis of mouse femur. The defect was a cylinder of $0.9 \mathrm{~mm}$ in diameter and $0.3-0.5 \mathrm{~mm}$ depth (Fig. $2 \mathrm{~b}$ ). No scaffold was used in this case and the bone healing was measured at 14 and 28 days using micro-CT. In order to model this in vivo experiment, we assumed that there are bone fluxes at the bottom and surrounding walls of the hole, and there is no flux from the top side. As the geometry and the boundary conditions were similar to our case, Equation (6) was used to calculate BVF in the defect.

\section{Results}

\subsection{In vivo results}

Fig. 3 shows the evolution of bone in the same location of a representative sample at different time points. Bone formation started as early as 2 weeks close to the bone-scaffold interface, as can be seen in region $R_{44}$ for instance. The interior of scaffold $\left(R_{11}\right)$ was left empty until almost 7 weeks had passed (Fig. 4). At later measurement times, the rate of bone formation in $\mathrm{R}_{44}$ decreased and BVF approached an asymptotic value $(C)$. The rate of bone formation in $\mathrm{R}_{11}$ seemed to be constant and BVF was still increasing at the later measurements.

\subsection{Model parameters identification}

The identification of the three parameters of our model allowed us to obtain a good fit of the in vivo data, both temporally and spatially (line red, Fig. 4). Not only was the estimated model within the confidence interval, but it also follows the pattern of BVF
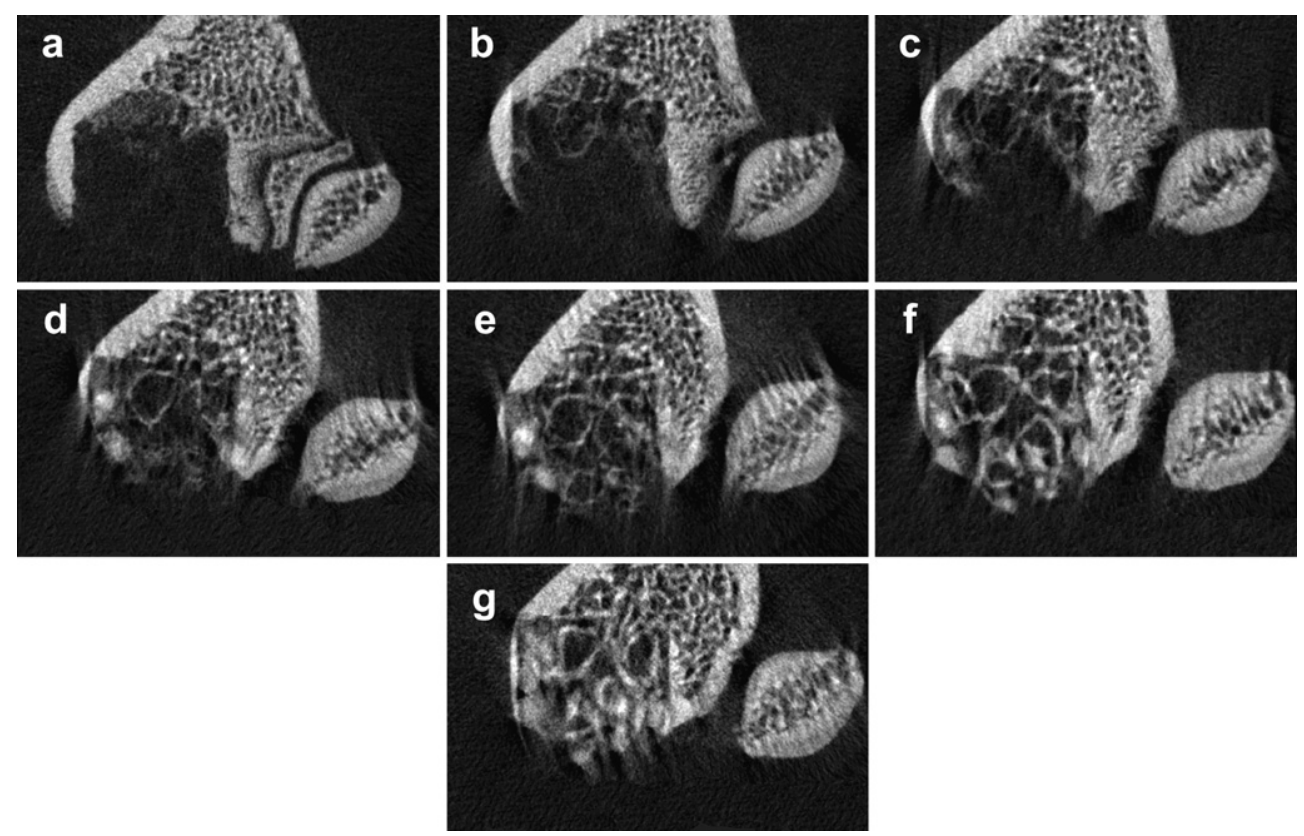

Fig. 3. Cross-section of scaffold and bone at different time points, (a) 2 weeks, (b) 4 weeks, (c) 7 weeks, (d) 11 weeks, (e) 15 weeks, (f) 22 weeks, and (g) 35 weeks, after the surgery. At early time points $(\mathrm{a}-\mathrm{c})$ we see small amount of bone formation at the peripheral part of the scaffold, and the interior of the scaffold is almost empty. However, at late time points $(\mathrm{f}-\mathrm{g})$, we observe almost a uniform distribution of bone. 
evolution over time in different regions. The estimated parameters and the corresponding 95\% confidence intervals are shown in Table 1.

\subsection{Validation of the model with the cases studied}

The results of the cases studied are shown in Table 2, comparing the experimental and predicted values for BVF. Although we used the same identified values of $C, \alpha$ and $h$ obtained from our rat experiment, the predicted values for BVF are close to the experimental values of the two published in vivo studies.

\subsection{Dimensional analysis}

Based upon the situation where BVF reaches $75 \%$ of the final BVF (C), we calculated the corresponding relation between Biot and Fourier dimensionless numbers for our in vivo experiment (Table 3 ) which is of bilinear form in the log-log scale (Fig. 5). In mathematical terms, this can be expressed as:

$\left\{\begin{array}{c}F o . B i=K_{1}, \quad \text { for } B i \ll 1 \\ F o=K_{2}, \quad \text { for } B i \gg 1\end{array}\right.$
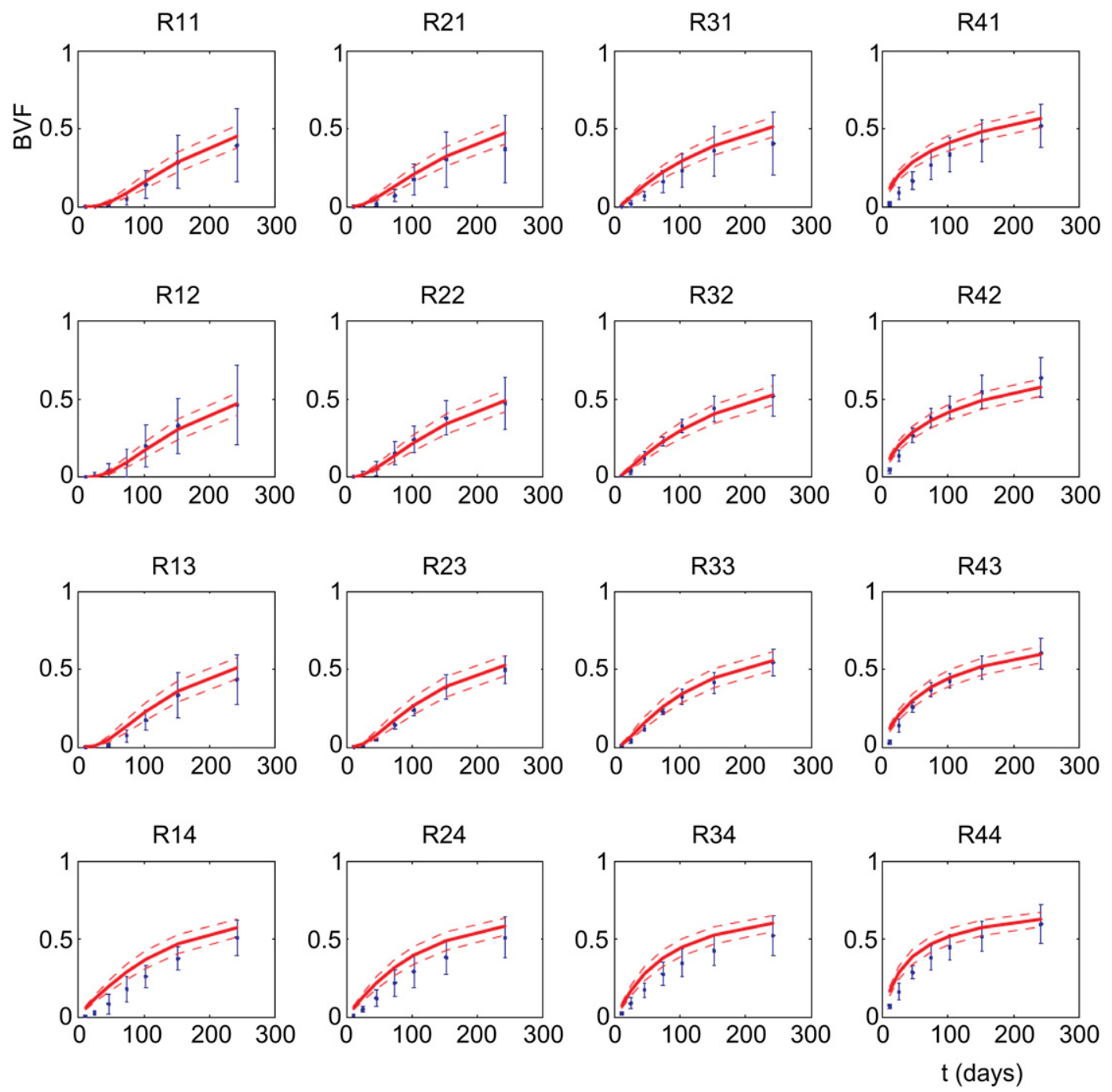

Fig. 4. The mean and its $95 \%$ confidence interval for BVF are shown as thin error bars for all regions inside the scaffold. The calculated value and the $95 \%$ confidence interval are shown as thick and dashed red curves, respectively. In almost all regions, the calculated interval is within the measured interval. (For interpretation of the references to colour in this figure legend, the reader is referred to the web version of this article.) 
Table 1

The estimated values of $C, \alpha, h$.

\begin{tabular}{llll}
\hline Parameter & Unit & Estimation & Confidence interval \\
\hline$C$ & $\%$ & 0.6859 & \pm 0.0329 \\
$\alpha$ & $m^{2} /$ day & 0.0041 & \pm 0.0005 \\
$h$ & $m m /$ day & 0.0073 & \pm 0.0012 \\
\hline
\end{tabular}

and regions. However, the model had only 3 unknown parameters that were identified using the in vivo data. The model could adequately fit the data and follow the pattern of bone formation at all time points and in every region. The in vivo data and the predicted curves showed two different patterns of evolution of BVF over time. In the regions further from bone, like $R_{11}$, we observed that curves are "s-shaped", meaning that there is a period of slow increase in rate of BVF (latency period) followed by a period of significant increase in BVF. In the regions closer to the bone, there is no latency period and the rate of BVF is initially high but then it decreases with time. One can expect that the larger the scaffold is, the longer the latency period in the inner part of scaffold will be.

Comparing the result of case studies with our model subjected our hypothesis to an additional test. In the case of the segmental defect of rat femurs, the predicted and experimental values were close, even though the scaffold used was PCL-TCP and the implantation site was different. This does not imply that every scaffold performs the same and all implantation sites have similar osteoinduction capabilities. However, PCL-TCP scaffold [23] had similar architecture to our scaffold and both were polymer-based and contained TCP particles. It is interesting to note that the characteristic time needed for the segmental defect to heal is more than 6 years. It confirms that the rat femoral model [23] is a critical size defect, i.e. it does not heal during the life time of the animal [25]. In the case of small perforations in mice femurs [24], our model resulted in BVF values close to the reported values, despite the fact that the animal model is different. Similar observations have been reported before in a bone chamber study where no significant difference was found between the bone ingrowth rate in rats and goats [26]. In a recent study, a contradictory result has been reported where it has been proposed that bone healing process is probably different between rat and sheep [27]. While this species dependency of the parameters is not by itself a limitation of the proposed model, generalization of identified parameters between species should be verified by experimental data.

The basic assumptions in our model were the governing law, i.e. the diffusion equation, and the type of boundary conditions. The reaction-diffusion model is the main equation in modeling cell migration [2], bone growth [28], bone healing [29], tumor growth [30], and angiogenesis [31]. In our study, we considered the case where the scaffold was not seeded with osteogenic cells or loaded with growth factors, hence we neglected the reaction term. As the boundary conditions, we assumed that the only source of bone formation is a flux of bone tissue at the bone-scaffold interface into the scaffold. Indeed, if the scaffold is seeded with osteogenic cells prior to implantation, the model could be extended into a reaction-

Table 2

Comparison between the experimental and predicted BVF for the three cases.

\begin{tabular}{lllc}
\hline In vivo experiment & $\begin{array}{l}\text { Time } \\
\text { points }\end{array}$ & $\begin{array}{l}\text { Experimental } \\
\text { BVF (\%) }\end{array}$ & $\begin{array}{l}\text { Predicted } \\
\text { BVF (\%) }\end{array}$ \\
\hline Rat distal femur (this & 7 Weeks & $19 \pm 2$ & 24 \\
article) & 22 Weeks & $41 \pm 7$ & 47 \\
Segmental defect in rat [23] & 3 Weeks & $1.4 \pm 0.2$ & 1.9 \\
& 12 Weeks & $4.2 \pm 1.0$ & 5.7 \\
Cortical perforation in & 14 Days & $45 \pm 13$ & 36 \\
mice [24] & 28 Days & $58 \pm 8$ & 52 \\
\hline
\end{tabular}

Table 3

Dimensionless numbers, characteristic time and length for the three in vivo cases. Although the Fourier number is the same for small cortical perforation model [24] and the large segmental defect [23], the characteristic time is massively different. Ten times change in the characteristic length between the two models results in 85 times change in the characteristic time.

\begin{tabular}{lllll}
\hline $\begin{array}{l}\text { In vivo } \\
\text { experiment }\end{array}$ & $\begin{array}{l}\text { Characteristic } \\
\text { length }(\mathrm{mm})\end{array}$ & $\begin{array}{l}\text { Biot } \\
\text { number }\end{array}$ & $\begin{array}{l}\text { Fourier } \\
\text { number }\end{array}$ & $\begin{array}{l}\text { Characteristic } \\
\text { time }\end{array}$ \\
\hline $\begin{array}{c}\text { Rat distal femur } \\
\text { (this study) }\end{array}$ & 1.5 & 2.67 & 0.40 & 31 Weeks \\
$\begin{array}{c}\text { Segmental defect } \\
\text { in rat [23] }\end{array}$ & 4 & 7.12 & 0.61 & 6.5 Years \\
$\begin{array}{c}\text { Cortical } \\
\text { perforation } \\
\text { in mice [24] }\end{array}$ & 0.4 & 0.80 & 0.60 & 4 Weeks \\
\hline
\end{tabular}

diffusion model by introducing a reaction term. The reaction term should then be identified with experimental data. In fact, the results of this study suggest that for larger scaffolds, one cannot rely only on the cells stemming from neighboring tissues, but an internal source of bone formation is needed. Although this is already known, the advantage of our model is its ability to predict the maximum size of scaffold that could be used without osteogenic factor while still supporting bone formation in a reasonable amount of time.

In this study, we introduced several new parameters. The scaffold osteoconduction coefficient, $\alpha$, is a property of scaffold and it depends on the material, surface properties, and architecture of scaffold. It also depends on the ease with which cells and other biological agents diffuse inside the scaffold. The peri-implant osteoinduction coefficient, $h$, is a property of the surrounding tissue and it depends on the species, age, site of implantation, vascularity of the surrounding tissue, and the quality of the interface between scaffold and bone [13]. The value of the peri-implant osteoinduction coefficient could then varied between different clinical situations. $C$ is defined as the final BVF in the scaffold. The value of $C$ depends on the architecture of the scaffold, particularly the porosity and surface area of the scaffold. If the scaffold is not porous enough, there is less room for forming bone. Also as bone

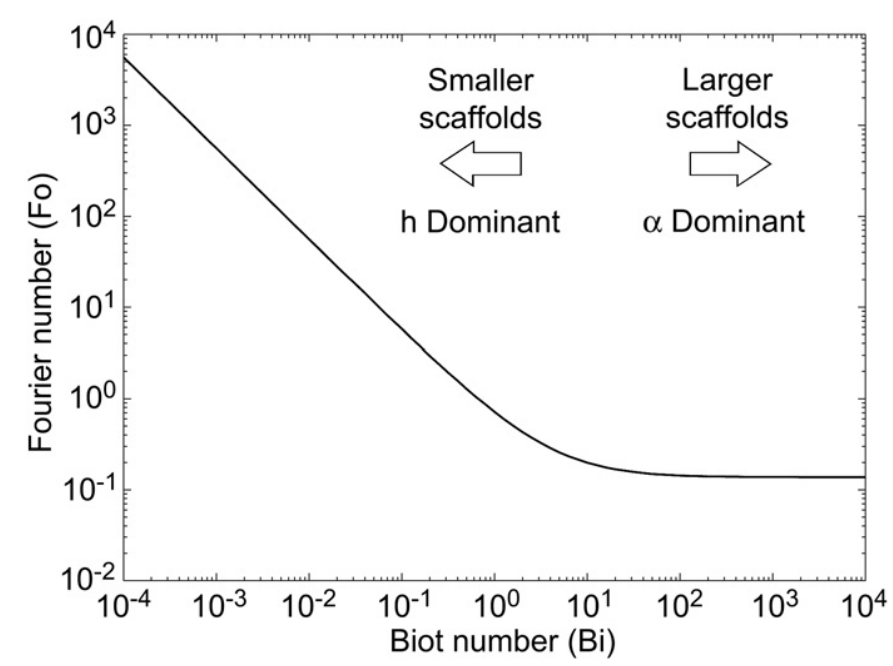

Fig. 5. The relation between Fourier and Biot numbers such that scaffold is filled with $75 \%$ of final $B V F, C$. The graph shows a clear bimodal behavior, where for low $B i$ values, there is an inverse relation between $B i$ and $F$, and for high $B i$ values, the $F o$ is constant. $h$ dominant means that scaffold does not play a key role in healing, while $\alpha$ dominant implies that if the scaffold is not osteoconductive enough (higher $\alpha$ ), osteogenesis is impaired. 
forms around the pore walls, we can expect to have higher BVF in scaffolds with a higher surface area.

The two dimensionless numbers, Biot and Fourier numbers, can be of special interest in the field of bone tissue engineering. Biot number indicates if bone formation is dominated by scaffold osteoconductivity, $\alpha$, or by the interaction with surrounding tissue, $h$. If $\alpha$ is larger than $h$ (i.e. the scaffold is highly osteoconductive), or if the size of the scaffold is small, then $B i<<1$. In this case, Equation (10) shows that the characteristic time, $T$, is proportional to the size of the scaffold and inversely proportional to $h$ (as $F o \cdot B i=h T / R=K 1$ ). Conversely, when $\alpha$ is much smaller than $h$ (i.e. the scaffold is poorly osteoconductive), or when the size of the scaffold is large, then $B i>>1$ and $T$ has a quadratic relation with the size of the scaffold such that $F_{0}=\alpha T / R^{2}=K_{2}$. In other words, when $B i<<1$ the scaffold does not play an important role in the bone regeneration process and the surrounding tissue will heal the defect easily. However, when $B i>>1$, the surrounding tissue will have difficulty healing the defect alone and the scaffold plays a key role in the healing process. The two cases studied support this concept very well. The femoral perforation model had a Biot number of 0.8 suggesting that the surrounding tissue could heal the defect even without the aid of any scaffold. In contrast, the segmental defect had a Biot number of 7.12, which is consistent with our suggestion that the scaffold is not sufficiently osteoconductive.

One important advantage of the proposed model is its simplicity. Our model was able to fit the quantitative in vivo data over a long period of time ( 35 weeks) and in different regions inside the scaffold. Furthermore, only three parameters, $C, \alpha$ and $h$, that are physically meaningful are required for the description. In contrast, available mathematical models are mostly mechanistic and they usually employ many parameters $[7,14,16]$. The estimation of additional parameters using only the BVF measurement is not possible due to the redundancy of the parameters. Nevertheless, mechanistic models give insights on the biological basis of osteogenesis inside the scaffold. A combination between the two approaches can further deepen our understanding of the process of bone formation.

Sengers et al. [2] studied MSC migration and proliferation on trabecular bone structure which is similar to the structure of our scaffold [21]. Employing a diffusion-reaction equation, they estimated the diffusion coefficient for cell migration to be $0.055 \mathrm{~mm}^{2}$ / day based on in vitro experimental results. Comparing this value to our estimated scaffold osteoconduction coefficient $\alpha$ (which can be regarded as diffusion of bone into scaffold, although bone does not literally diffuse), we see that bone formation is one order of magnitude slower than cell migration, i.e. the process of mineralization happens at a slower pace. This might suggest that the bottleneck in the process of bone formation is the differentiation and mineralization process, and not the cell migration.

When designing a strategy for bone tissue engineering, employing the proposed model can be helpful in several ways. First, it yields an estimate of the time required to mineralize implanted scaffolds. Thus, one can tailor the degradation rate of the scaffold to the actual rate of bone formation. Also, given the gradient of bone formation, one can estimate the evolution of mechanical properties of the scaffold-bone construct.

\section{Conclusions}

We hypothesized that bone formation inside scaffold follows diffusion phenomenon. We verified this hypothesis by a combined mathematical-experimental approach. The three parameters appearing in the diffusion law for bone formation characterize the final bone volume fraction, scaffold osteoconduction and the periscaffold osteoinduction properties. This model provides a direct theoretical tool to optimize the scaffold performance for bone formation. Interestingly, by introducing two dimensionless numbers in the diffusion equation, namely Biot and Fourier numbers, a comparison of the osteoconductive performances for different scaffolds at various implantation sites could also be obtained. The proposed model could successfully capture the course of bone formation in two other published in vivo experiments. Thus we conclude that bone formation inside scaffold can be predicted by diffusion law.

\section{Acknowledgments}

This project was supported by an SNSF grant (\#205320-121893) and the Inter-Institutional Center for Translational Biomechanics EPFL-CHUV-DAL.

\section{Appendix}

We start by nondimensionalizing the equations using

$u=\frac{c-C}{C}, x=\frac{r}{R}, y=\frac{z}{H}, \beta=\frac{R}{H}, F o=\frac{\alpha t}{R^{2}}, B i=\frac{h R}{\alpha}$

where $R$ is the radius of the scaffold and $H$ is the height of the scaffold. $B i$ and $F o$ are named similarly to the nomenclature of heat transfer [32]. Thus

$$
\begin{aligned}
& \frac{\partial u}{\partial F o}=\frac{\partial^{2} u}{\partial x^{2}}+\frac{1}{x} \frac{\partial u}{\partial x}+\beta^{2} \frac{\partial^{2} u}{\partial y^{2}} \\
& \left.\frac{\partial u}{\partial x}\right|_{x=1}=-\left.B i \cdot u(x, y, F o)\right|_{x=1} \\
& \left.\frac{\partial u}{\partial x}\right|_{x=0}=0
\end{aligned}
$$

$\left.\frac{\partial u}{\partial y}\right|_{y=1}=-\left.\frac{1}{\beta} B i \cdot u(x, y, F o)\right|_{y=1}$

$\left.\frac{\partial u}{\partial y}\right|_{y=0}=0$

$\left.u(x, y, F o)\right|_{F o=0}=-1$

Employing the method of separation of variables, we seek a solution of the form

$u(x, y, F o)=e^{-a F o} X(x) \cdot Y(y)$

Substituting (18) in (12), we obtain a representation in a variable separable form

$$
\begin{aligned}
& -a=\frac{X^{\prime \prime}}{X}+\frac{1 X^{\prime}}{x X}+\beta^{2} \frac{Y^{\prime \prime}}{Y} \\
& \text { Assuming } \\
& \frac{Y^{\prime \prime}}{Y}=-\omega^{2}
\end{aligned}
$$

and replacing it into (19) and re-writing it, we will have

$x^{2} X^{\prime \prime}+x X^{\prime}+\gamma^{2} x^{2} X=0$

where

$$
\gamma^{2}=a-\beta^{2} \omega^{2}
$$


Equation (21) can be identified as Bessel's differential equation of order zero, the solution of which takes the form

$X(x)=A J_{0}(\gamma x)+B Y_{0}(\gamma x)$

where $J_{n}(x)$ is the Bessel function of the first kind of order $n$ and $Y_{n}(x)$ is the Bessel function of the second kind of order $n$. As $Y_{0}(0)$ is not finite, we require $B=0$.

Solving Equation (20) and replacing it into Equation (18), we will have

$u(x, y, F o)=e^{-\left(\gamma^{2}+\beta^{2} \omega^{2}\right) F o} A J_{0}(\gamma x) \cdot[C \sin (\omega y)+D \cos (\omega y)]$

Boundary condition (14) is already satisfied $\left(J_{1}(0)=0\right)$, and satisfying (16) entails $C=0$. In order to satisfy (13)

$B i \cdot J_{0}(\gamma)=\gamma J_{1}(\gamma)$

which is satisfied at $\gamma_{i}(i=1,2,3, \ldots)$. Similarly, satisfying (15) will result in

$B i=\beta \omega \tan (\omega)$

which is satisfied at $\omega_{j}(i=1,2,3, \ldots)$. Therefore the solution (24) should be generalized to include all such values of $\gamma_{i}$ and $\omega_{j}$, and the summation of such solutions gives the final solution as

$u(x, y, F o)=\sum_{i=1}^{\infty} \sum_{j=1}^{\infty} E_{i j} J_{0}\left(\gamma_{i} x\right) \cos \left(\omega_{j} y\right) e^{-\left(\gamma_{i}^{2}+\beta^{2} \omega_{j}^{2}\right) F o}$

where $E_{i j}=A_{i} D_{j}$. Applying initial condition (17), we will have

$E_{i j}=-\frac{\int_{0}^{1} \int_{0}^{1} x J_{0}\left(\gamma_{i} x\right) \cos \left(\omega_{j} y\right) \mathrm{d} x \mathrm{~d} y}{\int_{0}^{1} \int_{0}^{1} x J_{0}^{2}\left(\gamma_{i} x\right) \cos ^{2}\left(\omega_{j} y\right) \mathrm{d} x \mathrm{~d} y}$

which can be further simplified to

$E_{i j}=-\frac{4}{\gamma_{i}} \cdot \frac{J_{1}\left(\gamma_{i}\right)}{J_{1}\left(\gamma_{i}\right)^{2}+J_{1}\left(\gamma_{i}\right)^{2}} \cdot \frac{\sin \left(\omega_{j}\right)}{\omega_{j}+\sin \left(\omega_{j}\right) \cos \left(\omega_{j}\right)}$

Substituting the original variables back into Equation (27), we will have

$c(r, z, t)=C\left[1-\sum_{i=1}^{\infty} \sum_{j=1}^{\infty} E_{i j} J_{0}\left(\frac{\gamma_{i}}{R} r\right) \cos \left(\frac{\omega_{j}}{H} z\right) e^{-\left(\gamma_{i}^{2}+\beta^{2} \omega_{j}^{2}\right)} \frac{\alpha}{R^{2}} t\right]$

which is the final solution of Equation (2). Given the BVF, $c$, we can calculate the bone volume inside each region, $R_{p q}$, shown in Fig. $1 \mathrm{~b}$. For that, we need to integrate $c(r, z, t)$ over the volume of each region, $V_{R p q}$. The result is

$\left.B V(t)\right|_{R_{p q}}=C\left[V_{R_{p q}}-\sum_{i=1}^{\infty} \sum_{j=1}^{\infty} E_{i j} I_{i j} e^{-\left(\gamma_{i}^{2}+\beta^{2} \omega_{j}^{2}\right)} \frac{\alpha}{R^{2}} t\right]$

where $I_{i j}$ is

$I_{i j}=\frac{2 \pi R H}{\gamma_{i} \omega_{j}} \cdot\left[r J_{1}\left(\frac{\gamma_{i}}{R} r\right)\right]_{r_{p}}^{r_{p+1}} \cdot\left[\sin \left(\frac{\omega_{j}}{H} z\right)\right]_{z_{q}}^{z_{q+1}}$

where $r_{p}$ and $z_{q}$ denote the coordinates of the boundaries of each region.

\section{References}

[1] Muschler GE, Nakamoto C, Griffith LG. Engineering principles of clinical cellbased tissue engineering. J Bone Jt Surg [Am] 2004;86A(7):1541-58.

[2] Sengers B, Please C, Taylor M, Oreffo R. Experimental-computational evaluation of human bone marrow stromal cell spreading on trabecular bone structures. Ann Biomed Eng 2009;37(6):1165-76.
[3] Mygind T, Stiehler M, Baatrup A, Li H, Zou X, Flyvbjerg A, et al. Mesenchymal stem cell ingrowth and differentiation on coralline hydroxyapatite scaffolds. Biomaterials 2007:28(6):1036-47.

[4] Laschke MW, Harder Y, Amon M, Martin I, Farhadi J, Ring A, et al. Angiogenesis in tissue engineering: breathing life into constructed tissue substitutes. Tissue Eng 2006;12(8):2093-104.

[5] Guldberg RE, Ballock RT, Boyan BD, Duvall CL, Lin ASP, Nagaraja S, et al Analyzing bone, blood vessels, and biomaterials with microcomputed tomography. IEEE Eng Med Biol Mag 2003;22(5):77-83.

[6] Cartmell S, Huynh K, Lin A, Nagaraja S, Guldberg R. Quantitative microcomputed tomography analysis of mineralization within three-dimensional scaffolds in vitro. J Biomed Mater Res A 2004;69(1):97-104.

[7] Bailon-Plaza A, van der Meulen $\mathrm{MCH}$. A mathematical framework to study the effects of growth factor influences on fracture healing. J Theor Biol 2001; 212(2):191-209.

[8] Karageorgiou V, Kaplan D. Porosity of 3D biomaterial scaffolds and osteogenesis. Biomaterials 2005;26(27):5474-91.

[9] Mastrogiacomo $M$, Scaglione S, Martinetti R, Dolcini L, Beltrame $F$ Cancedda $\mathrm{R}$, et al. Role of scaffold internal structure on in vivo bone formation in macroporous calcium phosphate bioceramics. Biomaterials 2006;27(17): 3230-7.

[10] Duty AO, Oest ME, Guldberg RE. Cyclic mechanical compression increases mineralization of cell-seeded polymer scaffolds in vivo. J Biomech Eng 2007; 129(4):531-9.

[11] Roshan-Ghias A, Terrier A, Bourban PE, Pioletti DP. In vivo cyclic loading as a potent stimulatory signal for bone formation inside tissue engineering scaffold. Eur Cell Mater 2010;19:41-9.

[12] Montjovent MO, Mark S, Mathieu L, Scaletta C, Scherberich A, Delabarde C et al. Human fetal bone cells associated with ceramic reinforced PLA scaffolds for tissue engineering. Bone 2008;42(3):554-64.

[13] Roshan-Ghias A, Pioletti DP. Surgical preparation of bone-scaffold interface is critical for bone regeneration inside tissue engineering scaffold. J Orthop Res 2011;29(5):767-72.

[14] Checa S, Prendergast PJ. Effect of cell seeding and mechanical loading on vascularization and tissue formation inside a scaffold: a mechano-biological model using a lattice approach to simulate cell activity. J Biomech 2010; 43(5):961-8.

[15] Sandino C, Planell JA, Lacroix D. A finite element study of mechanical stimuli in scaffolds for bone tissue engineering. J Biomech 2008;41(5):1005-14.

[16] Sanz-Herrera JA, Garcia-Aznar JM, Doblare M. A mathematical model for bone tissue regeneration inside a specific type of scaffold. Biomech Model Mechanobiol 2008;7(5):355-66.

[17] Amor N, Geris L, Vander Sloten J, Van Oosterwyck H. Computational modelling of biomaterial surface interactions with blood platelets and osteoblastic cells for the prediction of contact osteogenesis. Acta Biomat 2011;7(2): 779-90.

[18] Moreo P, García-Aznar JM, Doblaré M. Bone ingrowth on the surface of endosseous implants. Part 1: mathematical model. J TheorBiol 2009;260(1):1-12.

[19] Prendergast PJ, Huiskes R, Soballe K. ESB Research Award 1996. Biophysica stimuli on cells during tissue differentiation at implant interfaces. J Biomech 1997;30(6):539-48.

[20] Claes LE, Heigele CA. Magnitudes of local stress and strain along bony surfaces predict the course and type of fracture healing. J Biomech 1999;32(3): 255-66.

[21] Mathieu LM, Mueller TL, Bourban PE, Pioletti DP, Muller R, Manson JAE. Architecture and properties of anisotropic polymer composite scaffolds for bone tissue engineering. Biomaterials 2006;27(6):905-16.

[22] Seber GAF, Wild CJ. Computational methods for nonlinear least squares. John Wiley \& Sons, Inc.; 2005.

[23] Rai B, Oest ME, Dupont KM, Ho KH, Teoh SH, Guldberg RE. Combination of platelet-rich plasma with polycaprolactone-tricalcium phosphate scaffolds for segmental bone defect repair. J Biomed Mater Res A 2007;81(4) 888-99.

[24] Monfoulet L, Rabier B, Chassande O, Fricain J-C. Drilled hole defects in mouse femur as models of intramembranous cortical and cancellous bone regeneration. Calc Tissue Int;86(1):72-81.

[25] Jager M, Sager M, Lensing-Hohn S, Krauspe R. The critical size bony defect in a small animal for bone healing studies (II): implant evolution and surgical technique on a rat's femur. Biomediz Technik 2005;50(5):137-42.

[26] van der Donk S, Buma P, Aspenberg P, Schreurs BW. Similarity of bone ingrowth in rats and goats: a bone chamber study. Comp Med 2001;51(4): 336-40.

27] Checa S, Prendergast PJ, Duda G. Inter-species investigation of the mechanoregulation of bone healing: comparison of secondary bone healing in sheep and rat. J Biomech 2011;44(7):1237-45.

[28] Garzón-Alvarado D, García-Aznar J, Doblaré M. A reaction-diffusion model for long bones growth. Biomech Model Mechanobiol 2009;8(5):381-95.

[29] Adam JA. A simplified model of wound healing with particular reference to the critical size defect. Math Comp Model 1999;30(5-6):23-32.

[30] Jiang Y, Pjesivac-Grbovic J, Cantrell C, Freyer JP. A multiscale model for avascular tumor growth. Biophys J 2005;89(6):3884-94.

[31] Manoussaki D. A mechanochemical model of angiogenesis and vasculogenesis. Model Math Analyse Num 2003;37(4):581-99.

[32] Incropera F, Dewitt D, Bergman T, Lavine A. Fundamentals of heat and mass transfer: John Wiley \& Sons Inc 